\title{
ACCURACY ASSESSMENT FOR CAD MODELING OF FREEFORM SURFACE DESCRIBED BY EQUATION
}

\author{
Grzegorz GOLBA* \\ *Faculty of Mechanical Engineering, Department of Production Engineering, Bialystok University of Technology, \\ ul. Wiejska 45C, 15-351 Białystok, Poland \\ g.golba@doktoranci.pb.edu.pl
}

received 14 July 2014, revised 21 July 2015, accepted 22 July 2015

\begin{abstract}
This paper presents the results of comparative analysis of modeling accuracy the freeform surface constructed by using a variety of algorithms for surface modeling. Also determined the accuracy of mapping the theoretical freeform surface described by mathematical equation. To model surface objects used: SolidWorks 2012, CATIA v5 and Geomagic Studio 12. During the design process of CAD models were used: profile curves, fitting parametric surface and polygonal mesh. To assess the accuracy of the CAD models used Geomagic Qualify 12. On the basis of analyse defined the scope of application of each modeling techniques depending on the nature of the constructed object.
\end{abstract}

Key words: B-Spline, CAD Model, Cloud of Points, Reverse Engineering, NURBS, Surface Reconstruction, Triangulation

\section{INTRODUCTION}

Due to the higher requirements of constructed elements, designers are forced to construct products containing freeform surfaces. CAD models are used at all stages of the production process - from design to evaluation of the manufacturing accuracy. To investigate the manufacturing accuracy of the element or preparing technical documentation is carried out by process called digitalization. The aim of this process is obtaining a cloud of points which representing the geometry of the object. Scanning devices are different in structure, principle of operation, accuracy and the nature of the generated results. The measurement data may be gained by contact method using the coordinate measuring machine (CMM) or non-contact method using optical scanners, etc. CMM generates clouds with a small number of regularly located points. Non-contact scanners have worse measurement accuracy compared with $\mathrm{CMM}$, and the measurement results are represented by clouds with large numbers of irregularly located points. Taking into account the number of points in the cloud received by digitizing an object it is possible to apply different modeling techniques. In the case of modeling on a small number of regularly located points are used profile curves (Section 2.1). In the case of a cloud of points generated by non-contact scanning method cannot use this method for constructing the CAD model. For this reason, the process of preparing the CAD model is based on the generation of parametric surfaces on a cloud of points or a polygonal mesh (see section 2.2). Errors created in all these steps of manufacturing are included in the manufacturing accuracy. Required accuracy is directly related to the area of application of the element. The surfaces of optical components require the highest accuracy of manufacturing $\left(10^{-3}-10^{-6} \mathrm{~mm}\right)$. In the case of the most common applications, such as automotive, airplane, etc. the highest precision of manufacturing is smaller $\left(10^{-1}-10^{-3} \mathrm{~mm}\right)$ (Savio and De Chiffre, 2007).
Analyzing the available literature it can be concluded that, there are many publications which described the mathematical nature of curves (Hylewski, 2010; Piegl, 1989), and the parametrical surfaces used in freeform modeling (Hoffmann and JoanArinyo, 2002; Nyirenda et al., 2006; Paulos et al., 2008; Van den Berg et al., 2003). An important factor in the process of design models are a class of continuity used in design and styling of CAD surfaces (Putz, 2004). The process of modifying the curves and NURBS surfaces (Non-Uniform Rational B-Spline) by modifying the control points and weighting factors described in (Piegl and Tiller, 1997). More practical character has articles in which researchers describe the techniques used in the design of $C A D$ models (Hoffmann and Joan-Arinyo, 2002). In position (Van den Berg et al., 2002) described the process of parameterization, and attempt to invent a system for the recognition of freeform shapes. Construction digital models of scanned objects used in the process of reverse engineering de-scribed in (Gawlik and Wójcik, 2003; Hylewski, 2009; Kruth and Kersterns, 1998; Wróbel, 2010). Evaluation the accuracy of the reconstruction process containing the step of freeform surface modeling is described in (Werner, 2012).

In the available literature, there are no accuracy analysis of CAD modeling based on a cloud of points. For this reason is justified to try to compare modeling techniques which are presented in this article. The article presents the process of modeling the surface of irregular shape described by a mathematical formula enabling subsequent analysis of the accuracy of modeling, dependent of the type of design techniques. Were compared the three most popular freeform surface modeling techniques in CAD programs used to design or reverse engineering. The results of the analysis described in this article allows to choose the appropriate modeling techniques matched to a specific category of surface, taking into account the nature of its geometry, application and required accuracy of manufacturing. will be possible to initial estimate of modeling deviations occurring during the CAD 
model design resulting from the application of the techniques described in this article

\section{THEORETICAL BASIS}

Choosing the proper techniques of freeform surface modeling must take into account application, and hence the required manufacturing accuracy of the element (Savio and De Chiffre, 2007). Modeling procedure can result in errors, which values exceed the requirements established during manufacturing. In the case of modeling which is based in a cloud of points approach to the process of generate the CAD model may be different. It is determined by the number, and nature of the location of points in the cloud. In most engineering programs during the construction of CAD model on a cloud of points with a small number of regularly located points are used profile curves. For this purpose, the curves B-spline (Basis spline) forming profiles that are the backbone for future extrude of the parametric surface (Section 2.1). Profile curves interpolate points called nodes (points included in the curve) (Piegl and Tiller, 1997). B-spline Curves in case of modeling based on theoretical cloud of points are interpolate curves expressed using spline functions, i.e. functions defined by polynomials on a coherent sum of sub-intervals. The process of interpolation for parametric curves consists in determining the interpolation function within the range defined by the theoretical cloud of points. Searched function returns certain values at points which are nodes of curves (theoretical points in cloud). In other words, interpolation function passes through all the nodes, and the error of interpolation nodes must be unconditionally equal to 0 . Interpolation error can be reduced by increasing the number of nodes which are part of the curve. In order to improve approximations of interpolation function are used additional conditions of smoothness at the connection point of polynomials for ensuring the continuity of the second derivative of the spline function. In case of generate freeform surface based on cloud with a lot of irregularly located points can be used triangulation and parametric surfaces called NURBS (see section 2.2, 2.3). In the case of modeling using a triangle mesh is used approximation process of triangle mesh by the parametric surface. The task of approximation is to find the parametric surfaces that reflect as accurately as possible the nature of the modelled geometry. Looking for solutions close as possible to ideal during modeling freeform surface is used the method of least squares which minimizing the sum of squared errors occurring during the generation of the surface. In other words, this method fits surface to the data in the form of theoretical points or profile curves in such a way that the overall estimation error (for all points or curves) was as small as possible.

In the article the basis for modeling was a cloud of theoretical points generated by mathematical equation. In the case of the CAD model constructed using the profile curves, these points are as nodes. Then on the skeleton constructed by profile curves extruded a parametric surface.

\subsection{B-spline Curves}

This type of curves used in CAD modeling determines a string of control points enabling local control of the shape. The curve is determined by the interval parameter divided into sub-intervals called nodes. In the case of equidistant nodes curve is called uniform. The division of the range of the curve in a non-uniform way creates NURBS curve. The main advantage of B-spline curves is the ability to modify any part of the curve without affecting the shape of the remainder (Piegl, 1989). Thanks to these properties of B-spline curves it is possible to modeling profile curves for extrude the parametric surface. To designate any point on the curve B-spline is used the following equation (Piegl and Tiller, 1997):

$$
p(\mathrm{t})=\sum_{\mathrm{i}=0}^{\mathrm{m}-\mathrm{n}-1} p_{i} N_{i}^{n}(t) \text { for } t \in\left[\mu_{n}, \mu_{m-n}\right]
$$

where: $n$-degree of the curve, $m+1$ - number of nodes, $p_{i}-$ control points, $N_{i}^{n}(t)$ - normalized B-spline function of $n$ degree, $t$ - parameter of interval, $u_{n}, u_{m-n}$ - nodes of the curve.

\subsection{Triangulation}

Non-contact scanning techniques used in reverse engineering generate clouds with a huge number of irregularly located points. This creates a serious problem when editing a cloud of points consisting thousands of measurement points. The problem is caused by increase the main memory of the computer which needs during the process of constructing a CAD model. Many of specialist software which enables processing large amounts of data were created to prevent this problem. The process of constructing a CAD model has become simple, and completely automated through the use of triangulation. This process involves a connection of points in clouds by using the curves so that each point will be a vertex of the triangle. To make this possible a cloud of points is subjected to pre-treat to remove unwanted noise and distortion. In a subsequent step the number of points in areas with a small shape variation is reduces. Then, in order to obtain a uniform triangle mesh model is subjected to appropriate transformations, such as mesh simplification, editing and deleting an overlap of meshes.

The final step of the construction of a CAD model is to generate parametric surface on a triangular mesh. This process uses a global algorithm of approximation to fit the geometry of the parametric surface to the triangle mesh (Piegl and Tiller, 1997).

\subsection{NURBS Surfaces}

The NURBS surfaces are the most flexible, taking into account the design of CAD objects, because faithfully reflects the nature of freeform shapes. It is possible to extrude the surface on profile curves (approach applied to cloud with a small number of points). The second method generates the surface on a cloud of points or a triangular mesh (this method is used for clouds with large numbers of irregularly located points). Considering NURBS surfaces can be seen, that each modification of number, and position of control points in the control grid causes a local change in the shape of the generated surface (Piegl and Tiller, 1997).

The shape of B-spline surface is described by equation (2):

$B(u, v)=\sum_{i=0}^{n 1} \sum_{j=0}^{m} d_{i, j} N_{i, s}(u) N_{j, r}(v)$

where: $d_{i, j}$ - vertices of control grid, $N_{i, s}(u), N_{j, r}(v)$ - basis functions of B-spline curve.

In the case of the representation of the real object using parametric surfaces, points defining the geometry of the object should be given in homogeneous coordinate system. NURBS 


\section{ACCURACY ANALYSIS OF MODELING}

surface is generating by using internal mathematical tools to approximate the input data in the form of a cloud of points or triangular mesh. Approximation in this case approximates the cloud of points or triangular mesh by parametric surface.

\section{DESIGN OF CAD MODELS}

In studies of the accuracy of modeling were constructed three CAD models by using different techniques of modeling the freeform surface. The basis of the models was a cloud of theoretical points which was determined by equation (3), describing a mathematical model of the freeform surface. Generated 2600 points on the regular grid $X \times Y$ of size $1 \times 1 \mathrm{~mm}$ in range of $50 \times$ $50 \mathrm{~mm}$ (Fig. 2). Constructed CAD models compared with cloud of theoretical points described by mathematical equation (3).

$z=5 \cdot \sin \left(\frac{x}{12}\right)+\cos \left(\frac{y}{12}\right)$

where: $x-x$ - coordinate of point $x \in(0.50), y-y$-coordinate of point $y \in(0.50), z-z-$ coordinate of point.

Further in this chapter described three modeling techniques used while constructing CAD models of the surface at the theoretical cloud of points. The first model, which used a profile curves called MWPC. Other models were designed using: fitted parametrical surface (MWPS) and the triangular mesh (MWTM).

\subsection{Model with profile curves}

MWPC was constructed in SolidWorks using the B-spline curves run through a series of points in cloud. The process of generating curves was made accordance with the following scheme: all the points in a "0" row of cloud was combined with a single curve. Another row of points, that is, "1" row was left in the form of points, and the "2" row was also connected by means of the curve. This approach makes it possible to verify the accuracy of the modeling not only at the points belonging to the curve, but also at intermediate points. This allows determining the accuracy of approximation the changes of geometry in cloud of points by the parametric surface. In this manner generated 25 profile curves, every other row in the cloud of theoretical points. The nodes in curve were points belonging to the theoretical cloud of points. Therefore modeling errors, at all points belonging to the curves compared to the theoretical cloud of points should be 0 (Piegl, 1989). In a further step of modeling, profile curves were used in the process of create the parametric surface by extrude the profiles. MWPS was constructed in CATIA v5. On the theoretical cloud of points created parametric surface matched to the characteristics of the cloud. For this purpose was used the power fit option which contains the approximation by method of least squares to fit the shape of the surface to the cloud of points. MWTM was built in three stages. In the first stage, on the cloud of theoretical points was carried out the triangulation process, the result of which was triangular mesh (see section 2.2). Points in cloud were combined in segments in such a manner that each point of cloud has become a vertex of the triangle. In a second step was focused on modify, and remove the overlap of meshes in order to obtain a single model. In the last stage extruded the NURBS parametrical surface. The next stage of preparation for the analysis was to determine the reference component and test component.
Verification of the accuracy of the developed CAD models designed by using different modeling techniques was performed by using Geomagic Qualify 12. Using the procedure deviation determined the modeling errors between the reference object and test object. Before analysing, it was necessary to import the cloud of points and CAD models to the program. As a reference model adopted cloud of points and test object (subjected analysis) CAD models of the freeform surface.

\subsection{Analysis of the Accuracy of MWPC}

As first was conducted an analysis of the accuracy MWPC (Fig. 1).

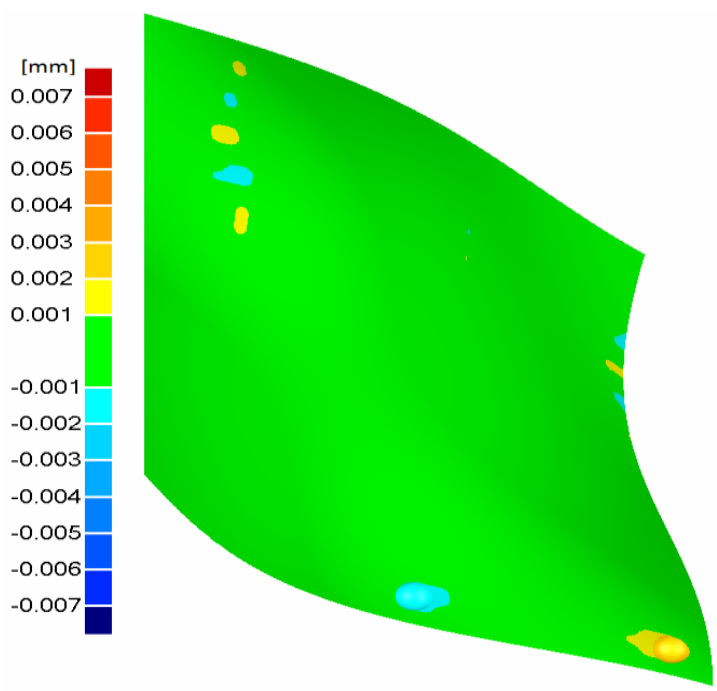

Fig. 1. Error map of MWPC

Analysing the map can be concluded that modeling errors are small. $99 \%$ of the analysed model points coincide with the theoretical cloud of points. This is because was used of interpolation curves which connecting the consecutive points during the process of constructing a CAD model (see sect. 2.1). The greatest value to the modeling error $(+0.001 \mathrm{~mm})$ appeared in the area of greatest slope surface.

Considering the percentage distribution of modeling errors of MWPC, it is clear that the percentage participation of errors with the same values, but with opposite signs is very close to each other (Fig. 2).

MWPC modeling technique except high-accuracy mapping contains one major drawback, which is the time-consuming process of constructing the profile curves passing through all the points in the cloud. This fact practically disqualifies this method of constructing in modeling of objects with large sizes for example reconstructions castles. Another disadvantage of this modeling technique is the inability to use of the data in the form of a cloud of points obtained by contactless scanning, where generated a large number of irregularly spaced points. In this case, during the construction of the profile curves program which was used to constructed CAD model would generate a huge demand in the operating memory of the computer. 


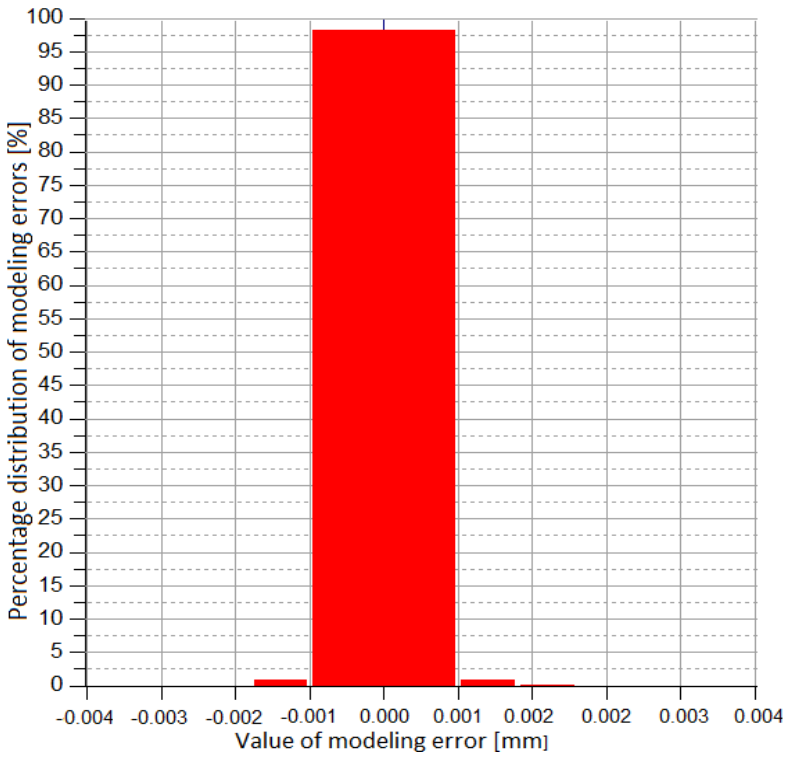

Fig. 2. Percentage distribution of errors MWPC

\subsection{Analysis of the Accuracy of MWPS}

Another model under review in terms of the accuracy of modeling was a MWPS (Fig. 3).

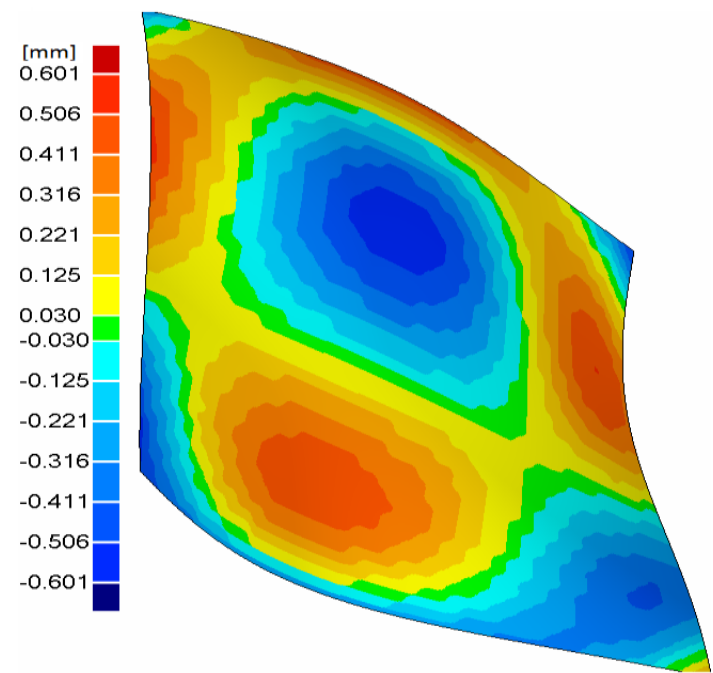

Fig. 3. Error map of MWPS

Large values of errors in MWPS, reaching of value \pm 0.601 $\mathrm{mm}$, occurred in the areas of the largest peaks and depressions, while the smallest errors occurred in the areas of change the curvature. This distribution modeling errors results from the fact that parametric surface which approximate the shape of theoretical cloud of points was used to generate CAD model. It introduces significant errors in the process of construction of a CAD object. Taking into account the distribution of errors MWPS can be concluded, that this technique is the best to modeling objects characterized by low volatility of shapes. Percentage distribution of errors MWPS clearly shows the considerable variation of the values and the percentage of occurrences (Fig. 4). Most errors (13.5\%) which were occurred during the construction of a CAD model reached a value of $+0.120 \mathrm{~mm}$, which indicate a low accuracy MWDP. Taking into account graph shown in figure 10 it can be concluded that this modeling technique is propose to be used in the design of large-scale CAD models of objects, where the modeling errors of $0.3 \mathrm{~mm}$ does not have a major impact on the final result of the project. Proper application of this modeling technique would be reconstruction of sculptures and castles, where inaccuracy of mapping object reaching of tenths part of a millimetre is the most acceptable.

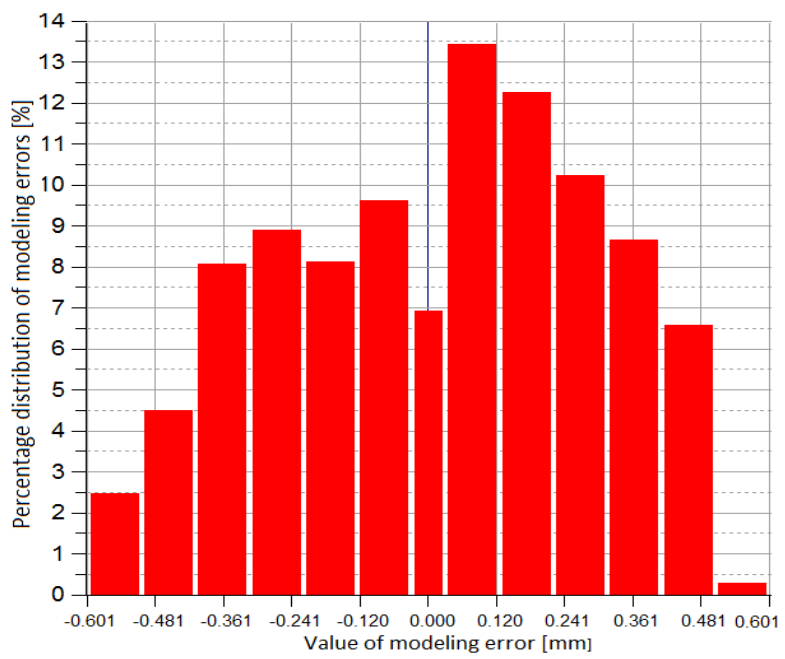

Fig. 4. Percentage distribution of errors MWPS

\subsection{Analysis of the accuracy MWTM}

Third analysis of accuracy was subjected MWTM (Fig. 5). Mesh of triangles made it impossible an accurate reconstruction of geometry the clouds of points in areas with significant elevations and depressions. Triangles are flat, so that an accurate representation of complex geometry, characterized by the freeform shape was not possible.

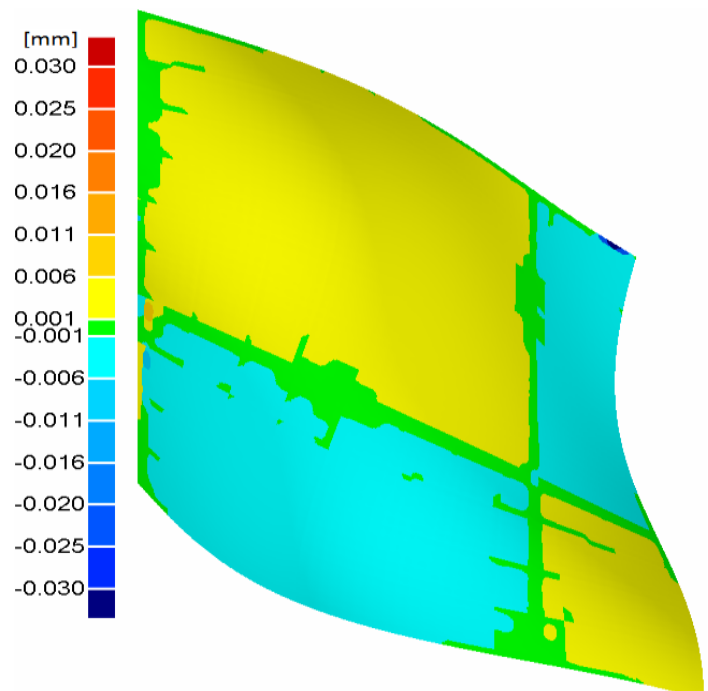

Fig. 5. Error map of MWTM

Errors which made during the process of generating a CAD model are result of using the parametric surfaces. NURBS surface is fitted to the surface of a triangle mesh, which as previously mentioned does not reflect all the changes shape. Percentage distribution of errors MWTM was shown in figure 6 . The largest 
percentage of instances (27\%) was recorded for the error value of $+0.006 \mathrm{~mm}$. The highest values of errors reaching $+0.030 \mathrm{~mm}$ occurred in $0.2 \%$ of the freeform surface. The accuracy of the mapping model is not as high as in the case of the model made MWPC technique. This results from the fact that the CAD model is the result of approximation process (generation of parametric surface) instead of interpolating (profile curves which goes through a points in cloud of points) as in the case MWPC model. Due to the possibility of easy processing of large amounts of data in the form of clouds with a large number of points this method is recommended for processing of data generated by noncontact digitization.

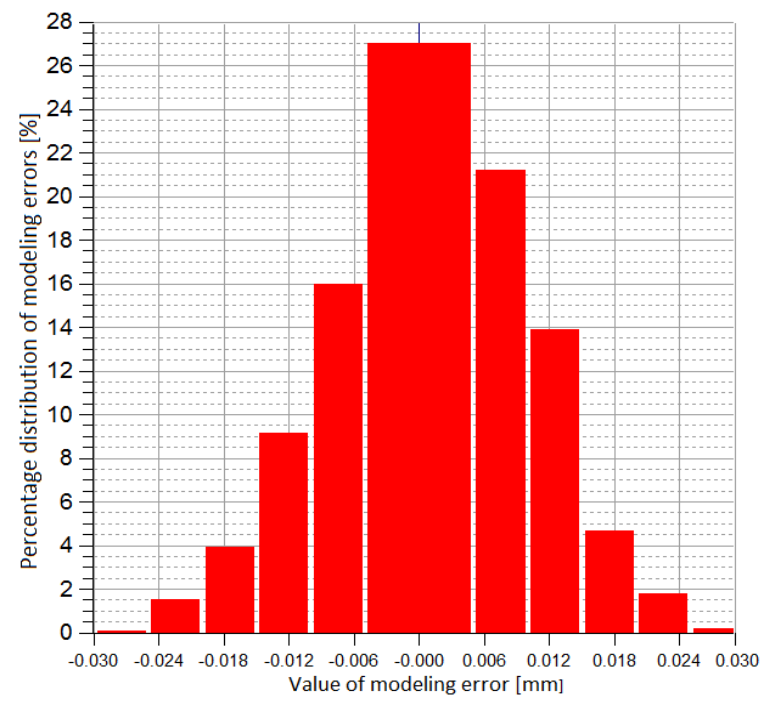

Fig. 6. Percentage distribution of errors of MWTM

\section{CONCLUSIONS}

Analysing the maximum modeling errors arising from the technique used to construct the CAD model can be seen a clear advantage in favour of the model MWPC. The maximum error values reach $+0.007 \mathrm{~mm}$, and are almost one hundred times smaller than those resulting from the application of modeling techniques MWPS $(+0.601 \mathrm{~mm})$. MWPC technique is suit for modeling the freeform surface, and the flat surface because of the high accuracy of mapping the shape of the cloud of points. In the case of design objects, in which the accuracy of the mapping is very important, MWPC modeling technique is most recommended. The only serious limitation in terms of use it is the difficulty, which appears when modeling is based on a cloud with a lot of points. This is caused by considerable demand of the CAD program in operational memory. The maximum modeling error $(+0.030 \mathrm{~mm})$ resulting from the application of MWTM modeling techniques is twenty times smaller than the modeling error of in MWPS. Taking this fact into account MWTM technique should be used to modeling objects with large dimensions, or in the case of constructing a CAD model based on a cloud with a very large number of irregularly spaced points. The highest accuracy of mapping the shape of the object by using this technique of modeling obtained in case of surface with a small variation of the shape. These results from the fact that the triangles are planar figures, which less illustrate the local variation of geometry. Taking into account the use of freeform surfaces, and hence the required precision of manufacturing (Savio and De Chiffre, 2007) can be analysed to evaluate the possible application of modeling techniques. Errors arising at the stage of digitalization, modeling and production are a part of manufacturing error, which should be taken into account in the selection of appropriate modeling techniques. MWPC technique can be successfully used for modeling small turbine blades, and automotive parts (acceptable manufacturing error $0.020 \mathrm{~mm}-0.001 \mathrm{~mm}$ ). MWTM is suitable for construction of objects such as aircraft components (maximum manufacturing error $0.500 \mathrm{~mm}-0.020 \mathrm{~mm}$ ). Castles elements, aircraft wings, large-scale sculptures and objects without large changes in the shape it is best to design using the technique MWPS.

Worth considering during the design elements with significant size containing complex surfaces is to use a combination of two modeling techniques. The MWPC technique will enable high accuracy in constructing the model with complex shape. MWTM technique could be applied in areas where local changes in the geometry of the shape are small.

\section{REFERENCES}

1. Gawlik J., Wójcik A. (2003), Modeling and assessing the accuracy of the freeform surfaces in the wmp-cad-cam system (in Polish), Prace Naukowe Instytutu Technologii Maszyn i Automatyzacji Politechniki Wrocławskiej, Vol. 84, 173-182.

2. Hoffmann CM., Joan-Arinyo R. (2002), Parametric modeling, In: Farin G, Hoschek J,Kim MS (editors), Handbook of CAGD, Elsevier, 519-541.

3. Hylewski D. (2009), Representation of curves in CAD systems Bezier curves (in Polish), Prace Naukowe Katedry Budowy Maszyn Politechniki Śląskiej, Vol. 1, 273-282.

4. Hylewski D. (2012), Attempt to verify the method for selecting the parameters of digitizing the freeform surface for the purpose of reverse engineering in Catia v5 system (in Polish), Inżynieria Maszyn, Vol.3, 128-146.

5. Kruth JP., Kersterns A. (1998), Reverse engineering modeling of free-form surface from point clouds subject to boundary condition, Journal of Materials Processing Technology, Vol. 76, 120-127.

6. Nyirenda PJ., Mulbagal M., Bronsvoort WF. (2006), Definition of freeform surface feature classes, Computer-Aided Design and Applications, Vol. 3(5), 665-674.

7. Paulos J., Nyirenda., Willem F. Bronsvoort. (2008), Numeric and curve parameters for freeform surface feature models, ComputerAided Design, Vol. 40 (8), 839-851.

8. Piegl L. (1989), Modifying of rational B-spline. Part 1: Curves, Computer Aided Design, Vol. 21, 509-518.

9. Piegl L., Tiller W. (1997), The NURBS book, 2nd ed., Springer, New York.

10. Putz B. (2004), From G0 to G3 - class of smoothness in the design and styling surface (in Polish), Mechanik, Vol. 11, 759-764.

11. Savio E., De Chiffre L. (2007), Metrology of freeform shaped parts, CIRP Annals - Manufacturing Technology, Vol. 56 (2), 810-835.

12. Van den Berg E., Bronsvoort W. F., Vergeest J. S. M. (2002), Freeform feature modeling: concepts and prospecs, Computers in Industry, Vol. 49 (2), 217-233.

13. Van den Berg E., Van der Meiden H. A., Bronsvoort W. F. (2003), Specification of freeform features. In: Elber G, Shapiro V (editors), Proceedings of the eighth ACM symposium on solid modeling and applications, New York: ACM Press, 56-64.

14. Werner A. (2012), Assessment of accuracy in the process of reverse engineering the spatial object (in Polish), Pomiary, Automatyka, Robotyka, Vol. 5, 86-90.

15. Wróbel I. (2010), Reverse engineering as a method of creating digital models of objects with complex shapes (in Polish), Mechanik, Vol. 3, 212-214. 